\title{
Silicon-on-Insulator Nanophotonic Waveguide Circuit for Fiber-to-the-Home Transceivers
}

\author{
D. Vermeulen(1), G. Roelkens(1), J. Brouckaert(1), D. Van Thourhout(1), R. Baets(1) \\ R. Duijn(2), E. Pluk(2), G. Van den Hoven(2) \\ 1: Photonics Research Group, Ghent University-IMEC, Sint-Pietersnieuwstraat 41, B-9000 Ghent - Belgium \\ e-mail: Diedrik.Vermeulen@Ugent.be \\ 2: GENEXIS B.V., Lodewijkstraat 1a, 5652 AC Eindhoven, The Netherlands \\ e-mail: r.duijn@genexis.nl
}

\begin{abstract}
\section{Introduction}

Silicon-on-insulator technology has many advantages for the realization of photonic integrated circuits. First of all the platform is CMOS-compatible and due to the high refractive index contrast, the designs are very compact. Furthermore, out-of-plane coupling can be realized by the use of a diffractive grating [1]. This makes wafer scale testing feasible. By unifying these advantages it is possible to fabricate nanophotonic integrated circuits in large volumes at a low cost.
\end{abstract}

We present a SOI nanophotonic waveguide circuit for FTTH transceivers. A grating duplexer and planar concave grating are combined to couple and demultiplex the 1310, 1490 and 1550nm communication channels.

Point-to-point Fiber-to-the-Home optical access networks require large volume and low-cost optical transceivers, both at the subscriber and the central office side. From the perspective of the transceiver at the subscriber side, $1310 \mathrm{~nm}$ is the upstream channel and $1490 \mathrm{~nm}$ and $1550 \mathrm{~nm}$ are the downstream channels for data and CATV. The passive optical part of a fiber-to-the-home transceiver has to couple and demultiplex these three communication channels.

In this paper, we report on the design and fabrication of a nanophotonic waveguide circuit realizing this optical coupling and demultiplexing. With a grating duplexer [2] the light is coupled into the chip and the $1310 \mathrm{~nm}$ upstream channel is spatially separated from the downstream channels.

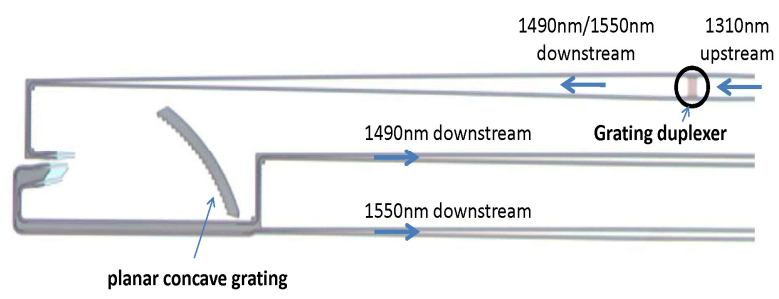

Figure 1: Microscope image of a grating duplexer in combination with a Planar Concave Grating (PCG) demultiplexer for FTTH applications

With a planar concave grating demultiplexer [3], the downstream channels $1490 \mathrm{~nm}$ and $1550 \mathrm{~nm}$ are splitted and the $1310 \mathrm{~nm}$ wavelength channel is filtered to reduce the crosstalk levels. A microscope image of the fabricated structure is shown in Figure 1.
This circuit is fabricated using standard CMOS technology, on a silicon wafer with a $220 \mathrm{~nm}$ thick silicon waveguide core and a $2 \mu \mathrm{m}$ thick buried oxide layer.

\section{Grating Duplexer}

In order to couple and at the same time split the upstream and downstream wavelength bands, we use a 1-dimensional grating duplexer. The working principle is shown in Figure 2. Under a certain angle of the optical fiber, the Bragg condition is fulfilled for both wavelengths $\lambda_{1}$ and $\lambda_{2}$ and the 2 wavelength bands will couple in opposite directions. The grating period, duty cycle and number of periods have been optimized using a particle swarm optimization algorithm, in order to achieve maximum coupling efficiency for both the upstream and downstream wavelength bands.

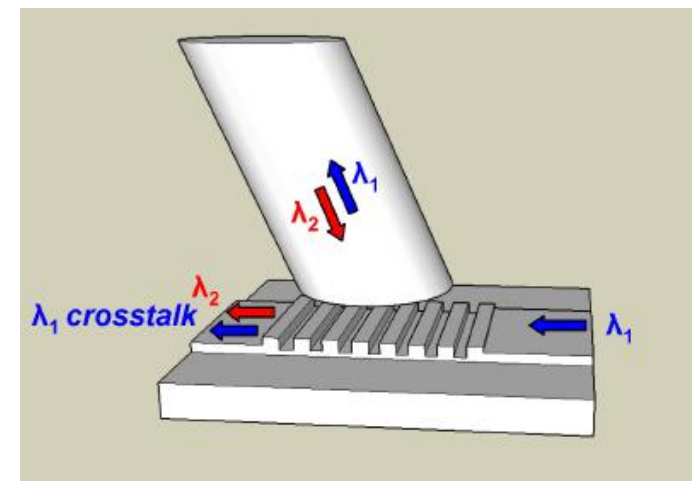

Figure 2: Operation principle of a grating duplexer. $\lambda_{1}$ represents the upstream wavelength band, $\lambda_{2}$ represents the downstream wavelength band.

The duplexer has been designed in a way that the upstream band around $1310 \mathrm{~nm}$ is coupled in the forward direction and the downstream band around $1520 \mathrm{~nm}$ is coupled backwards. The measurement results of the grating duplexer are plotted in Figure 3. The period of the grating is $520 \mathrm{~nm}$ and the grating duty cycle is $40 \%$. The etch depth is $70 \mathrm{~nm}$. The number of grating periods is 20 . Index matching fluid was applied between the optical fiber facet and the 
grating duplexer to avoid reflections at the fiber facets and the fiber was tilted under an angle of 10 degrees. Standard single mode fiber was used for the experiments. The experiments were performed using TE polarized light. Making the grating duplexer polarisation independent can be done by using a two dimensional grating structure [2].
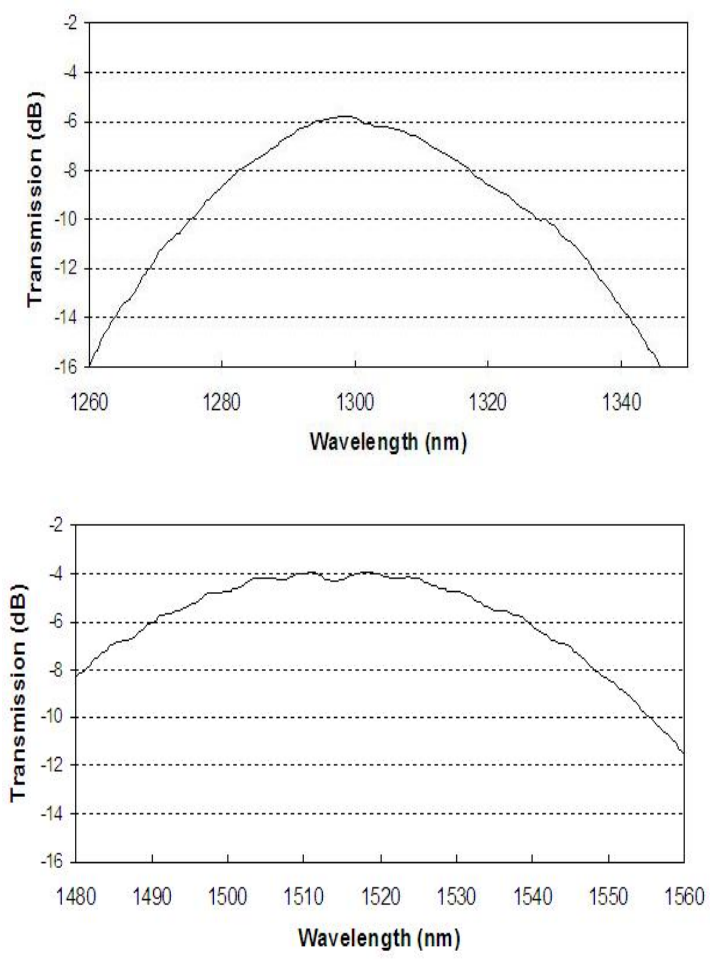

Figure 3: Transmission spectrum of the grating duplexer for the upstream band (upper graph) and the downstream band (lower graph)

The coupling efficiencies of the central wavelengths are $-6 \mathrm{~dB}$ for $1300 \mathrm{~nm}$ and $-4 \mathrm{~dB}$ for $1520 \mathrm{~nm}$. At the communication wavelengths 1310,1490 and 1550 $\mathrm{nm}$ the coupling efficiencies are respectively $-7 \mathrm{~dB},-6$ $\mathrm{dB}$ and $-8 \mathrm{~dB}$. Decreasing these losses can be done by first depositing an extra silicon layer prior to defining the grating [4]. Simulations show that efficiencies of $-1.9 \mathrm{~dB}$ are possible for both central wavelengths.

\section{Planar Concave Grating Demultiplexer}

Demultiplexing the downstream band is accomplished by using a planar concave grating demultiplexer (PCG) [3]. In order to avoid possible crosstalk from the grating duplexer as shown in figure 2, we also have to filter the $1310 \mathrm{~nm}$ wavelength in both downstream channels. This was done by adjusting the free spectral range of the grating demultiplexer. Measurements of the grating demultiplexer are shown in Figure 4.

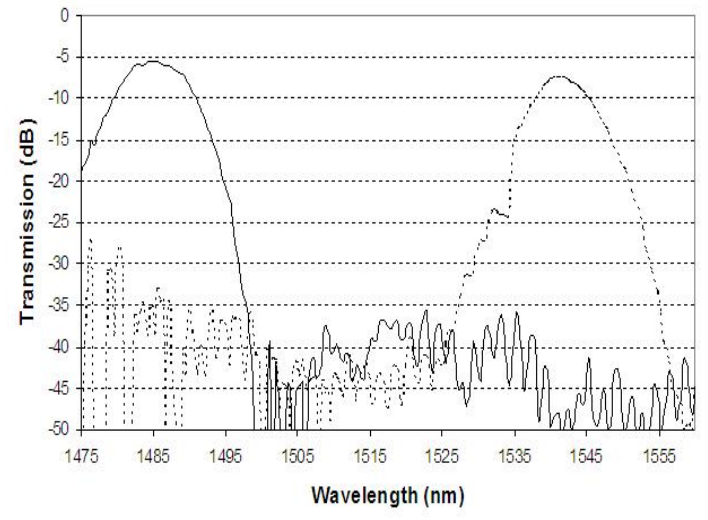

Figure 4: Transmission spectrum of the planar concave grating demultiplexer

The maxima of the transmission efficiencies are -5.4 $\mathrm{dB}$ for $1485 \mathrm{~nm}$ and $-7.6 \mathrm{~dB}$ for $1542 \mathrm{~nm}$ and are limited by the Fresnel reflection at the Silicon/Air interface. The crosstalk at these maxima is about -30 $\mathrm{dB}$ and $-40 \mathrm{~dB}$ respectively. Filtering of the $1310 \mathrm{~nm}$ channel is as good as $-40 \mathrm{~dB}$. Several techniques to reduce the insertion loss of this demultiplexer exist; for example with high reflectivity Bragg reflectors, as described in [5], the insertion loss can be reduced by $4 \mathrm{~dB}$.

\section{Conclusions}

We have demonstrated how to couple, demultiplex and filter $1310 \mathrm{~nm}, 1490 \mathrm{~nm}$ and $1550 \mathrm{~nm}$ with the use of just 2 integrated optical components. A grating duplexer couples the light and splits the upstream/downstream bands. The PCG separates the $1490 \mathrm{~nm}$ and $1550 \mathrm{~nm}$ while filtering $1310 \mathrm{~nm}$. In addition we have suggested how to increase the performance of these components and make the device polarisation independent. The proposed configuration contains all functionalities for the passive optical part of an SOI FTTH transceiver, while exploiting all advantages of the silicon-on-insulator platform. We believe that this proof of concept is a stepping stone for future low cost SOI fiber-to-thehome transceiver chips.

\section{References}

1. D. Taillaert et al IEEE Photonics Technology Letters, 15 (2003), pages 1249-1251

2. G. Roelkens et al Optics Express, 15 (2007), pages 10091-10096

3. J. Brouckaert et al Journal Of Lightwave Technology, 25 (2007), pages 1269-1275

4. G. Roelkens et al Applied Physics Letters, 92 (2008), page 131101

5. J. Brouckaert et al IEEE Photonics Technology Letters, 20 (2008), pages 309-311 